\title{
Supporting Information: A Direct Comparison of Three Buckling-Based Methods to Measure the Elastic Modulus of Nanobiocomposite Thin Films
}

\author{
Taylor C.Stimpson ${ }^{1}$, Daniel A. Osorio ${ }^{2}$,Emily D. Cranston ${ }^{2,3 *}$, Jose M. Moran-Mirabal ${ }^{4 *}$
}

${ }^{1}$ Department of Chemical Engineering, McMaster University, 1280 Main Street West, Hamilton, ON L8S 4L7, Canada

${ }^{2}$ Department of Chemical and Biological Engineering, The University of British Columbia, 2360 East Mall, Vancouver, BC V6T 1Z3, Canada

32Department of Wood Science, The University of British Columbia, 2424 Main Mall, Vancouver, BC, V6T 1Z4, Canada, and Department of Chemical and Biological Engineering, The University of British Columbia, 2360 East Mall, Vancouver, BC V6T 1Z3, Canada

${ }^{4}$ Department of Chemistry and Chemical Biology, McMaster University, 1280 Main Street West, Hamilton, ON L8S 4M1, Canada

* Corresponding authors:

mirabj@mcmaster.ca

emily.cranston@ubc.ca 


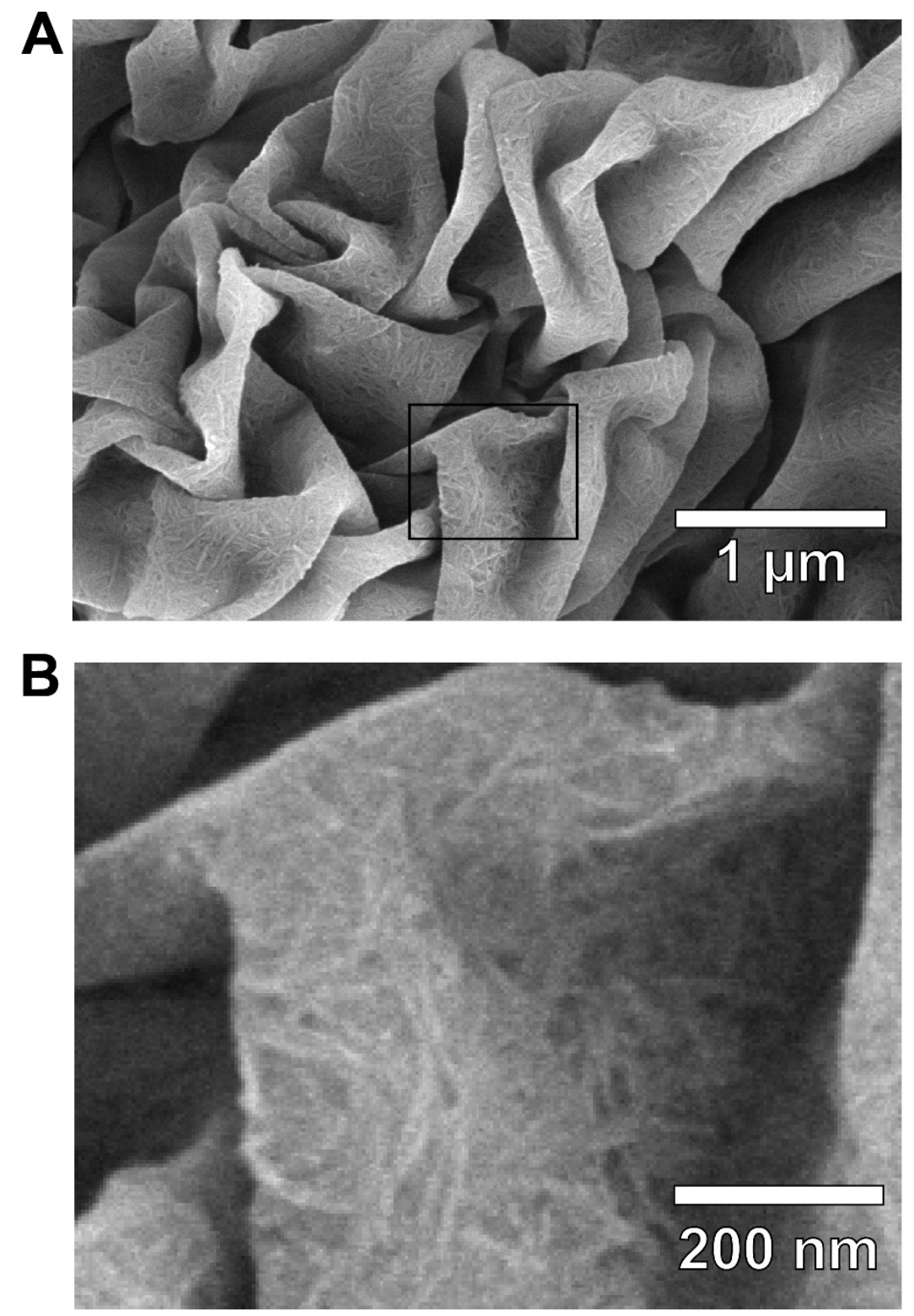

Figure S1. A) SEM image of a $1 \mathrm{bL} \mathrm{CNC}_{3.0}-\mathrm{PEI}_{0.1}$ film, buckled via biaxial thermal shrinking, with B) an enlarged view of the region outlined in the black square in A). The image was obtained by Christine Lancelon-Pin of CERMAV-CNRS in Grénoble, France. 
Table S1. Summary of relevant parameters output from statistical analysis of buckling methods (paired t-test and ANOVA). Paired t-tests were performed between pairs of methods for each CNC-PEI composition, and for pairs of CNC-PEI compositions for each method. ANOVA was performed between methods for a single CNC-PEI composition, and between CNC-PEI compositions for each individual method. $\mathrm{P}$ value statistic is shown $\times 10^{2}$ to show relevant significant digits.

\begin{tabular}{|c|c|c|c|c|}
\hline & Paired t-Test & & & \\
\hline $\begin{array}{l}\text { CNC }_{0.03-} \\
\text { PEI }_{0.1}\end{array}$ & Uniaxial to Biaxial & Biaxial to SIEBIMM & $\begin{array}{l}\text { Uniaxial to } \\
\text { SIEBIMM }\end{array}$ & $\begin{array}{l}\text { 1-way ANOVA } \\
\text { (between groups) }\end{array}$ \\
\hline $\mathrm{d}_{\mathrm{f}}$ & 2 & - & - & - \\
\hline$P$ value & 0.019 & - & - & - \\
\hline t value & 7.24 & - & - & - \\
\hline F value & - & - & - & - \\
\hline $\begin{array}{l}\text { Critical F } \\
\text { value }\end{array}$ & - & - & - & - \\
\hline $\begin{array}{l}\mathrm{CNC}_{3.0^{-}} \\
\mathrm{PEI}_{0.1}\end{array}$ & Uniaxial to Biaxial & Biaxial to SIEBIMM & $\begin{array}{l}\text { Uniaxial to } \\
\text { SIEBIMM }\end{array}$ & $\begin{array}{l}\text { 1-way ANOVA } \\
\text { (between groups) }\end{array}$ \\
\hline$\overline{d_{f}}$ & 2 & 2 & 2 & 2 \\
\hline$P$ value & 0.17 & 0.40 & 0.23 & 0.63 \\
\hline t value & 2.08 & -1.06 & -1.69 & - \\
\hline$F$ value & - & - & - & 0.48 \\
\hline $\begin{array}{l}\text { Critical F } \\
\text { value }\end{array}$ & - & - & - & 3.98 \\
\hline $\begin{array}{l}\text { CNC }_{3.0-} \\
\text { PEI1.0 }\end{array}$ & Uniaxial to Biaxial & Biaxial to SIEBIMM & $\begin{array}{l}\text { Uniaxial to } \\
\text { SIEBIMM }\end{array}$ & $\begin{array}{l}\text { 1-way ANOVA } \\
\text { (between groups) }\end{array}$ \\
\hline $\mathrm{d}_{\mathrm{f}}$ & 2 & 2 & 2 & 2 \\
\hline$P$ value & 0.02 & 0.33 & 0.52 & 0.45 \\
\hline $\mathrm{t}$ value & -69.80 & 1.29 & 0.78 & - \\
\hline F value & - & - & - & 0.85 \\
\hline $\begin{array}{l}\text { Critical F } \\
\text { value }\end{array}$ & - & - & - & 3.98 \\
\hline
\end{tabular}




\begin{tabular}{|c|c|c|c|c|}
\hline Biaxial & $\begin{array}{l}\text { CNC }_{0.03} \text { PEI }_{0.1} \text { to } \\
\text { CNC } 3.0-\text { PEI }_{0.1}\end{array}$ & $\begin{array}{l}\text { CNC }_{0.03}-\text { PEI }_{0.1} \text { to } \\
\text { CNC } 3.0-\text { PEI }_{1.0}\end{array}$ & $\begin{array}{l}\text { CNC }_{3.0} \text {-PEI } 0.1 \text { to } \\
\text { CNC }_{3.0} \text {-PEI } 1.0\end{array}$ & $\begin{array}{l}\text { 1-way ANOVA } \\
\text { (between groups) }\end{array}$ \\
\hline $\mathrm{d}_{\mathrm{f}}$ & 2 & 2 & 2 & 2 \\
\hline$P$ value & 0.0031 & 0.014 & 0.24 & 0.11 \\
\hline $\mathrm{t}$ value & -17.80 & -8.39 & -1.67 & - \\
\hline F value & - & - & - & 2.74 \\
\hline $\begin{array}{l}\text { Critical F } \\
\text { value }\end{array}$ & - & - & - & 3.89 \\
\hline Uniaxial & $\begin{array}{l}\text { CNC }_{0.03}-\text { PEI }_{0.1} \text { to } \\
\text { CNC }_{3.0}-\text { PEI }_{0.1}\end{array}$ & $\begin{array}{l}\text { CNC }_{0.03}-\text { PEI }_{0.1} \text { to } \\
\text { CNC }_{3.0}-\text { PEI }_{1.0}\end{array}$ & $\begin{array}{l}\text { CNC }_{3.0}-\text { PEI }_{0.1} \text { to } \\
\text { CNC }_{3.0}-\text { PEI }_{1.0}\end{array}$ & $\begin{array}{l}\text { 1-way ANOVA } \\
\text { (between groups) }\end{array}$ \\
\hline $\mathrm{d}_{\mathrm{f}}$ & 2 & 2 & 2 & 2 \\
\hline$P$ value & 0.0030 & 0.0018 & 0.0005 & 0.039 \\
\hline $\mathrm{t}$ value & -18.32 & -23.70 & -42.90 & - \\
\hline F value & - & - & - & 4.31 \\
\hline $\begin{array}{l}\text { Critical } F \\
\text { value }\end{array}$ & - & - & - & 3.89 \\
\hline SIEBIMM & $\begin{array}{l}\mathrm{CNC}_{0.03}-\mathrm{PEI}_{0.1} \text { to } \\
\mathrm{CNC}_{3.0}-\mathrm{PEI}_{0.1}\end{array}$ & $\begin{array}{l}\mathrm{CNC}_{0.03}-\mathrm{PEI}_{0.1} \text { to } \\
\text { CNC }_{3.0}-\mathrm{PEI}_{1.0}\end{array}$ & $\begin{array}{l}\text { CNC }_{3.0}-\text { PEI }_{0.1} \text { to } \\
\text { CNC }_{3.0}-\text { PEI }_{1.0}\end{array}$ & $\begin{array}{l}\text { 1-way ANOVA } \\
\text { (between groups) }\end{array}$ \\
\hline $\mathrm{d}_{\mathrm{f}}$ & - & - & 2 & - \\
\hline$P$ value & - & - & 0.49 & - \\
\hline $\mathrm{t}$ value & - & - & 0.85 & - \\
\hline F value & - & - & - & - \\
\hline $\begin{array}{l}\text { Critical F } \\
\text { value }\end{array}$ & - & - & - & - \\
\hline
\end{tabular}

